\title{
Dichoptic induction of movement aftereffects contingent on color and on orientation
}

\author{
M. J. POTTS and J. P. HARRIS \\ Brain and Perception Laboratory, Department of Anatomy, Medical School, University of Bristol, \\ Bristol, BS8 ITD, England
}

\begin{abstract}
Some comparative experiments on the dichoptic induction of the movement aftereffect (MAE) contingent on color and the MAE contingent on orientation are reported. Colorcontingent movement aftereffects could be evoked only when the eye which had viewed color during adaptation also viewed color during test sessions. When the apparent color of the test field was changed by binocular color rivalry, contingent movement aftereffects (CMAEs) appropriate to the suppressed color were reported. After dichoptic induction of the orientationcontingent MAE, aftereffects could be obtained whether the eliciting gratings and stationary test fields were presented together to either eye alone or were dichoptically viewed.
\end{abstract}

Coltheart (1973) and Over, Long, and Lovegrove (1973) independently suggested that the failure of colored aftereffects contingent on orientation (McCollough, 1965) to transfer interocularly implied that the underlying mechanisms, specific for both color and orientation, had an exclusively (or predominantly) monocular site. Several later studies have called this attractively simple idea into question. First, Mackay and Mackay $(1973,1975)$ raised a prima facie difficulty by showing that the McCollough effect could be induced dichoptically, by viewing alternating homogeneous colored fields with one eye and simultaneously alternating achromatic gratings with the other. [The failure of Over et al. (1973) to demonstrate this effect was probably due to their comparatively brief periods of adaptation and their relatively insensitive methods of measurement.] The Mackays also noted that the direction of the effect depended on which eye viewed the grating. If this was the eye which had viewed color during adaptation ("color eye"), the aftereffects were negative, while viewing with the other ("pattern") eye yielded positive aftereffects, which the Mackays termed "anomalous." Mikaelian (1975), who adapted one eye to the usual alternating colored gratings while the other viewed achromatic gratings of the same orientation and spatial frequency, obtained effects similar to those of the Mackays from the color- and noncolor-adapted eyes. Secondly, Vidyasagar (1976), using an experimental procedure in which periods of binocular adaptation alternated with cancelling monocular adaptation, has reported evidence for a binocular McCollough effect. Following such an adaptation regime, binocularly viewed gratings yield normal, negative, colored CAEs, while

We thank the MRC, from whom M. J. P. received a research scholarship, for support. monocular viewing produces reports of positive (or anomalous) effects.

A further class of study, into which this paper falls, has tried to determine whether such effectswhich require some kind of interaction between the monocular pathways-are unique to the McCollough effect. Murch (1974) reported that the color-contingent MAE (Favreau, Emerson, \& Corballis, 1972; Mayhew \& Anstis, 1972) could be dichoptically induced, his stationary colored test field yielding negative CMAEs when presented to the color-adapted eye, but no reports (or inconsistent reports) of apparent movement when presented to the movement-adapted eye. In our first experiment, we tried to replicate this result for three reasons. The first concerns his experimental procedure. It is clearly important that, during adaptation, the eye viewing the colored field should be prevented from seeing the moving field, and vice versa. The method used by Murch was that of crossed Polaroid filters between the color eye and the moving field. One of these filters was mounted in a spectacle frame worn by the subject. If linear polarizing filters were used, any slight lateral head tilt would enable the color eye to see at least some movement. It would not then be surprising if that eye yielded a weak color-contingent MAE on test, but one would not be justified in concluding that it had been dichoptically induced. Secondly, we felt that the movementadapted eye deserved a closer look. The Mackays and Mikaelian both report positive McCollough effects from an eye which had adapted solely to achromatic patterns. One might therefore expect to find positive MAEs contingent on color when testing the movement eye after dichoptic adaptation to color and movement. Thirdly, it seemed possible that, with some suitable variations in test procedure, the dichoptic adaptation technique could be used to examine the question of whether the conscious perception of test color is 
necessary to evoke a color-contingent MAE. In a second experiment, we looked for the movement analogue of the kind of interocular generalization reported for the McCollough effect by Mikaelian (1975). A third experiment examined the dichoptic induction of the MAE contingent on the orientation of gratings. Because the simple aftereffects of tilt and of motion show interocular transfer in normals (e.g., Campbell \& Maffei, 1971; Wade, 1976), we expected, after dichoptic induction, to elicit orientationcontingent MAEs monocularly, through either eye, as well as dichoptically. In previous work (Potts \& Harris, 1975), it was found that this CMAE, discovered by Mayhew and Anstis (1972), transferred interocularly. As well as demonstrating the uniqueness of the binocular asymmetries of CAEs involving color, we hoped that this experiment would provide a yardstick against which to judge the results with color-contingent MAEs, since in some test conditions the latter might be very weak.

\section{GENERAL METHOD}

\section{Apparatus}

A schematic plan of the basic apparatus is shown in Figure la. The observer $(0)$ sat with his chin on a rest, facing a white $22^{\circ}$ $\times 15^{\circ}$ screen $(\mathrm{A})$, at a distance of $160 \mathrm{~cm}$. The screen had a $6^{\circ}$-diam circular hole cut in its center to accommodate a frosted perspex (Plexiglas) back-projection screen (B). A circular, clear perspex disk (D), approximately $35 \%$ of whose area was covered with randomly placed circular 1-min-diam black dots, was mounted on pulleys immediately behind this screen. It could be rotated

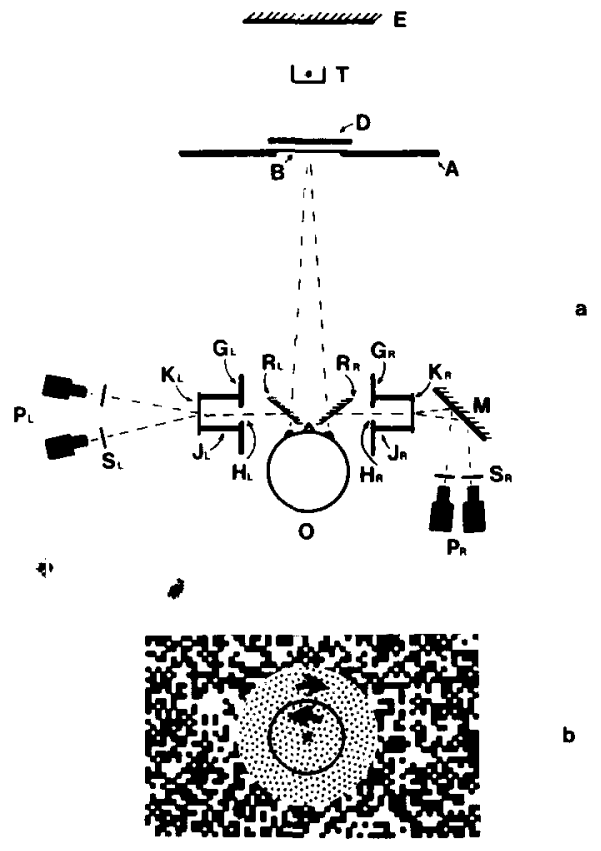

Figure 1.(a) Schematic plan of the apparatus (not to scale). See text for details. (b) Subjects' view of the display on Screens $A$ and $B$ (not to scale). During adaptation, the directions of movement of the counterrotating fields were reversed as the color or orientation of the other simultaneously presented stimuli was changed. through one of its pulleys at $3 \mathrm{rpm}$ by a synchronous motor whose direction could be reversed every $10 \mathrm{sec}$. Light from a 100 -W tungsten halogen point source $(T)$ was reflected by a plane mirror (E) to produce a shadow projection of the rotating dots on the screen. A counterrotating annulus (inside diameter, $6^{\circ}$; outside diameter, $10^{\circ}$ ) of similar random dots, surrounding the shadow-projected display, was added to the screen (A) using a slide projector (not shown), with a high-contrast black-and-white transparency, appropriately blanked off, rotating in its slide plane. This was edge driven by a reversible stepping motor, and its speed was adjusted to match that of the shadow-projected display. Neutral density filters were used to obtain a brightness match with the shadow display. It was hoped that this split-field display would enhance any CMAEs, as well as provide a control for any conditioned torsional eye movements. A second slide projector (again not shown in Figure 1) was used to project a stationary pattern of random dot texture (Julesz, 1971), which surrounded the moving fields, on the screen (A). (MAEs are more vivid if viewed against stationary landmarks-Anstis, 1961; Day \& Strelow, 1971). A small black fixation cross was provided at the center of the moving field. Figure $1 \mathrm{~b}$ shows the resulting display.

A reflecting surface $\left(R_{L}, R_{R}\right)$ could be mounted immediately in front of each eye, at $45^{\circ}$ to the subjects' line of sight. These were interchangeably either fully reflecting mirrors or $50 / 50$ beam splitters ("mixing cubes"). Two matt black opaque screens $\left(G_{L}, G_{R}\right)$ were mounted so that their circular apertures $\left(H_{L}, H_{R}\right)$ subtended the same visual angle $\left(10^{\circ}\right)$ as the outer diameter of the rotating fields. Two short circular black tunnels $\left(J_{L}, J_{R}\right)$ terminated in translucent (Ozatex) back-projection screens $\left(K_{L} K_{R}\right)$. Two pairs of slide projectors $\left(P_{L}, P_{R}\right)$, whose beams could be switched in and out (as the motor driving disk, $\mathrm{D}$, reversed its direction) by solenoid-operated shutters $\left(S_{L}, S_{R}\right)$, were used to project homogeneous colored patches or high-contrast gratings onto these screens. Because of limited space, the plane mirror $(M)$ was used to keep the projector/screen distance the same for both eyes. Lenses at $H_{L}, H_{R}$ were used to make the projected displays at $K_{L}, K_{R}$ parfocal with that at $B$.

The square-wave gratings used in these experiments were produced by black-and-white transparencies. Their spatial frequency was about 1 cycle/deg. Their dark bars had a luminance of about $7 \mathrm{~cd} / \mathrm{m}^{2}$ and their white bars, about $55 \mathrm{~cd} / \mathrm{m}^{2}$, from readings with an S.E.I. photometer. The colored fields were produced by Kodak Wratten filters, No. 26 (red) and No. 61 (green), the filters used by Murch (1974). Their percentage transmission, measured with a U.D.T. photocell (PIN 10AP), whose response closely approximates the CIE photopic luminosity curve, was very nearly equal. The luminances of the projected patches were matched, using this photocell, at about $60 \mathrm{~cd} / \mathrm{m}^{2}$.

The bright area of the moving fields had a luminance of about $50 \mathrm{~cd} / \mathrm{m}^{2}$ and the dark spots a luminance of about $5 \mathrm{~cd} / \mathrm{m}^{2}$. The apparatus was adjusted for each subject so that he saw, either monocularly or dichoptically, a circular field of color or grating, of the same size as, and superimposed on, the circular counterrotating dotted fields.

\section{Typical Procedure}

The same general type of procedure was used in all experiments, and will be outlined here, though variations from it will be indicated where appropriate in the reports of individual experiments. To adapt for dichoptically induced CMAEs, the following procedure was adopted: the right eye looked through a beam splitter, whose side faces were occluded with black card, at the rotating fields. A fully reflecting mirror was mounted in front of the other eye and adjusted so that the display at $K_{L}$ appeared binocularly superimposed on the moving field. Gratings of orthogonal orientation or colored fields were alternately projected to $K_{L}$ as the direction of rotation of the moving fields changed. Thus, a subject might adapt to clockwise (CW) center, counterclockwise (CCW) surround rotation in the right eye plus a superimposed red field in the left, alternating with $\mathrm{CCW}$ center, $\mathrm{CW}$ surround rotation in the right eye plus green in the left. Adaptation was completed by several quick alternations of the stimuli to cancel any simple 
MAEs from the final adapting direction of motion, and the moving display was then stopped.

Several different test conditions could then be employed with a suitable arrangement of mirrors and beam splitters; these will be described in the reports of individual experiments. These test conditions were run in a different order for each subject, and were usually given more than once, in blocks of some six alternations of the test fields. Testing each subject took about $20 \mathrm{~min}$ and began immediately after adaptation. In all test conditions, the subjects reported the direction (CW or $\mathrm{CCW}$ ) of any apparent movement of the stationary test field. The color or orientation of the displays at $K_{L}$ and $K_{R}$ was changed by the experimenter a few seconds after the reported end of any apparent movement. Although MAEs contingent on color and on orientation are very durable and can be elicited over a long period after the end of adaptation, each individual aftereffect is usually brief, and may last for only a few seconds or less. Thus, it is difficult to use techniques like matching or nulling to quantify such effects. We therefore used the duration of the CMAE as a measure of its strength. [Moulden (1974) has shown that the duration of a simple MAE is linearly related to its velocity, as measured by a matching technique; we assume this relationship to hold for CMAEs also]. In addition, when effects were weak, subjects rated their judgments as "certain", or "uncertain."

Test sessions were recorded on audio tape. The click produced by the solenoids as the display was changed acted as a time marker for the start of each CMAE. The tapes were later transcribed by the authors using a stopwatch to measure the interval between a solenoid click and the reported end of any apparent movement, when this exceeded $1 \mathrm{sec}$. In other cases, CMAEs were simply classified as "brief," and the subjects' rating of "certain" or "uncertain" noted.

Each report about the direction of apparent movement following an alternation of the fields in each test condition was classified as one of three types: "negative" (in the direction opposite to the movement paired with a particular color or orientation during adaptation), "positive"' (in the same direction), or "zero" (reported absence of apparent movement). Both naive and experienced observers of CAEs were used as subjects, since, it could be argued, the former might fail to detect weak effects, while the latter, if they knew or guessed the experimental hypothesis, might give false positive results. No systematic differences between these groups were found.

Subjects, who wore their usual optical corrections, were informally screened for color vision and stereopsis deficiencies, using Ishihara plates and the red/green anaglyphs of decreasing correlation published by Julesz (1971). None of the subjects used here had detectable defects of color vision, and all could correctly report depth in stereograms of $80 \%$ correlation.

\section{EXPERIMENT 1}

This experiment was an attempt to replicate Murch's (1974) findings on the dichoptic induction of CMAEs, though with some additional dichoptic test conditions. Some observations were also made when the apparent color of the test field was changed by binocular color rivalry.

\section{Procedure}

During adaptation, as shown in Figure 2, the subject's right eye viewed the rotating display, while his left eye viewed homogeneous color fields at $K_{L}$, via a fully reflecting mirror at $R_{L}$. Thus, his right eye could see a circular split moving field of black dots on a white ground (with a stationary surround of random black and white texture), while his left could see only a superimposed circular colored field with a homogeneous dark surround. Seven subjects, including the authors, were used. Five of them were experienced observers of CAEs, and all of them adapted for $20 \mathrm{~min}$.

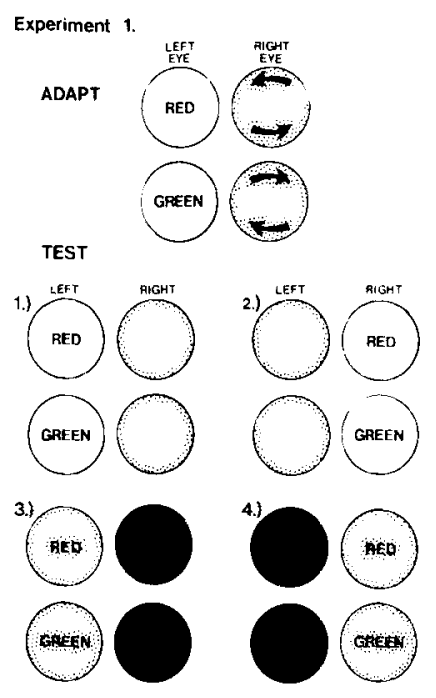

Figure 2. Diagram of the adaptation and test conditions of Experiment 1. During adaptation and each test condition, the upper pair of dichoptically presented stimuli alternated with the lower pair. Key to the four test conditions: (1) test as adapt, (2) test opposite to adapt, (3) test monocularly color eye, (4) test monocularly motion eye. See text for further details.

All subjects received four kinds of test. They were tested as they had adapted, by simply stopping the rotary movement and alternating the colored fields to the left eye, or in the opposite-to-adapt mode, with the color and test fields switched between the eyes. The two other tests were those described by Murch: They observed the test field monocularly, with either the color or the movement eye, through colored filters placed close to the eye and switched between red and green by a solenoid.

\section{Results and Discussion}

The proportions of the three types of response (negative, positive, zero) for each of the four test conditions are shown in Figure 3. Also shown is the mean duration of any reported apparent movement when this was sufficiently long to escape categorization as brief (B), together with the total number of responses $(\mathbf{N})$ in each test condition. In addition, where applicable, histogram bars are subdivided into "certain" (solid black) and "uncertain" (dotted) responses.

The results may be summarized as follows: When tested dichoptically as they had adapted, all subjects consistently reported CMAEs which were negative with respect to the adapting pairing of color and movement. These CMAEs were described as "powerful" and "unambiguous," with a mean duration of about $4 \mathrm{sec}$, though individual blocks of trials immediately or shortly after adaptation produced durations of up to $8 \mathrm{sec}$. In contrast, when the colored and stationary test fields were reversed to the eyes, reports of apparent movement were rare, were rated as "uncertain," and were equally divided between positive and negative. When the stationary test field was viewed monocularly through colored filters with the color eye, aftereffects were less readily obtained than they were in the dichoptic test-as-adapt condition, some $40 \%$ 


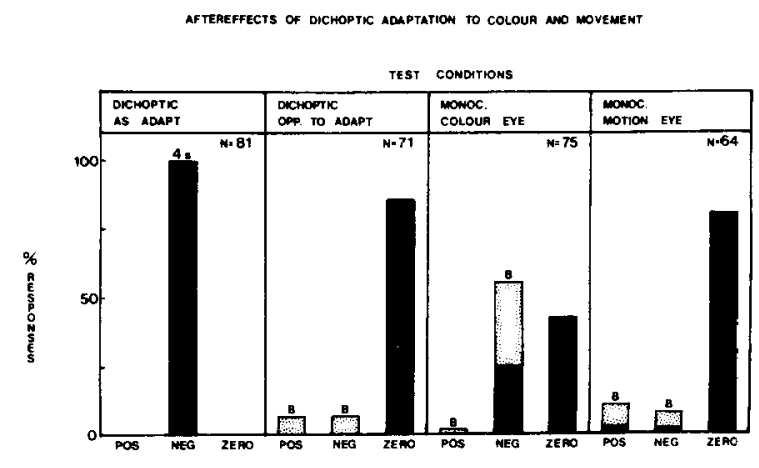

Figure 3. Reports from seven subjects of apparent movement of the stationary test field after dichoptic adaptation to alternating colored and moving fields. Bars indicate what percentage of the total number of responses $(N)$ in each test condition were of positive (POS), negative (NEG), or zero apparent movement. Mean durations of apparent movement are shown if this exceeded about 1 sec; otherwise they are shown as brief' (B). Solid black parts of bars indicate certain, and dotted parts uncertain responses. See text for further details.

of the trials producing no reports of apparent movement. However, when apparent movement was reported, it was consistently "negative," though generally brief, and on about half the trials it was rated as "uncertain." Viewing with the movement eye produced results very like those in the dichoptic opposite-to-adapt condition. Reports of apparent movement rarely occurred, and the proportions of "negative" and "positive" reports were similar.

Thus we have replicated and extended Murch's (1974) finding that the MAE contingent on color can be dichoptically induced. Our result is also consistent with the Mackays' $(1973,1975)$ report that negative McCollough effects can be obtained from the color eye after dichoptic adaptation. But their finding of a positive or anomalous McCollough effect from the pattern eye suggests that one might expect positive CMAEs from the movement eye in our experiment. Like Murch, we found no evidence of such an effect.

\section{Further Observations}

Our objection to Murch's dichoptic experiment was that one could not be sure that movement and color had been completely segregated at each eye. Because of this, an extra control test was run on four of our seven subjects in the dichoptic test-as-adapt condition. Though powerful CMAEs had been reported in this condition, we wondered whether they might have been produced by scattered colored light reaching the movement eye during adaptation and testing. As a check on this, the subjects were instructed to close their left (color) eyes during testing, while the colored fields projected to them were alternated. No CMAEs were then reported, though any scattered light would still have been reaching the right (movement) eye. This seemed to confirm our view that the obtained CMAEs had in fact been dichoptically induced.
A variant of the dichoptic test procedure also enabled us to make some observations relevant to the problem of the site of the adaptation underlying colorcontingent MAEs. We consistently found, after dichoptic adaptation, that the aftereffects from the dichoptic test-as-adapt condition were negative. Thus (ignoring for the purpose of exposition half the adapting and test cycle and the split field nature of the display), if a subject had adapted to a red field with his left eye and achromatic $\mathrm{CW}$ motion with his right, he reported apparent $\mathrm{CCW}$ movement of the stationary test field presented to his right eye, when his left eye was seeing the red field. We then interposed a green colored filter between his right eye and the test field. Because the eyes were now seeing different colors, color rivalry occurred. Sometimes the test field appeared green, sometimes red, and sometimes a patchy mixture of the two colors. The question then asked was: Does the apparent $\mathrm{CCW}$ movement (the CMAE) stop when the apparent color of the test field is green, or does the red field still seen by the color eye, though suppressed by color rivalry, continue to drive it? The answers of four subjects, who had each adapted dichoptically for at least $20 \mathrm{~min}$ and were tested on all possible color/eye combinations, were consistent. CMAEs negative with respect to the color seen by the color eye were almost always reported. On many occasions, these CMAEs, lasting for $5 \mathrm{sec}$ or more, persisted through several reversals of apparent color of the test field.

The failure of rivalry suppression to disrupt the CMAE is consistent with the finding of Lehmkuhle and Fox (1975) and Wade and Wenderoth (1978) that induction of the simple movement and tilt aftereffects, respectively, is unaffected by suppression. White, Petry, Riggs, and Miller (1978) have shown that the strength of McCollough effects from monocular adaptation is the same whether or not the gratings were suppressed during adaptation by achromatic stimuli seen by the other eye. Our results show that binocular suppression of the eliciting color does not inhibit the CMAE during testing.

These observations suggest two things. First, as already indicated, conscious perception of the eliciting color is not necessary to evoke a CMAE. Secondly, it appears that the binocular interaction of two monocular color channels-which must underlie binocular color rivalry-is independent of, or perhaps preceded by, the binocular interaction of the color information from one eye and the stationary pattern information from the other which must underlie the dichoptically induced CMAE.

It could be argued that, although the color from the motion eye often appeared to totally suppress the color from the color eye during testing, some color information from the nominally suppressed eye was leaking through to conscious perception and thus continuing to drive the aftereffect. If this was the case, one might have expected the apparent speed and spatial extent of 
the CMAE to change as the color eye became more or less dominant. However, no such effect was observed.

A second potential objection to our interpretation is that positive CMAEs from the motion eye indistinguishably alternated with negative CMAEs from the color eye. However, this account then has to explain why we found no positive CMAEs when the motion eye alone was tested.

\section{EXPERIMENT 2}

It is possible that our failure to obtain CMAEs in some conditions in Experiment 1 arose because our adapting stimuli were not optimal. Mikaelian (1975) reported that interocular generalization of the McCollough effect is found if the normally unadapted eye simultaneously views alternating achromatic gratings of the same spatial frequency and orientation as the colored gratings seen by the adapting eye. We looked at the effects of adapting to the movement analogue of this arguably more potent stimulus: while one eye inspected the black-and-white moving fields, the other viewed the same patterns through colored filters.

\section{Procedure}

The moving split field described above was used, and was binocularly viewed. The beam-splitting and projection equipment shown in Figure 1 was not used in this experiment. Colored filters over one eye were changed by a solenoid, as the patterned fields reversed their directions of movement. Five subjects, including the authors, each adapted for $20 \mathrm{~min}$.

When the moving fields had been stopped, the following test conditions were used: test binocularly, as adapt; test binocularly with color filters over the other eye; test color-plus-movement eye alone through color filters; test movement eye alone through color filters. The adapting and test conditions are shown schematically in Figure 4.

\section{Results and Discussion}

Figure 5 shows the results, classified on the same criteria as in Experiment 1, with whose results there are several similarities. Testing dichoptically as during adaptation or monocularly with the color-plusmovement eye yielded consistent reports of negative aftereffects with mean durations of 2 and $1.6 \mathrm{sec}$, respectively. (This kind of relationship would be expected if there were an additional dichoptic effect present in the test-as-adapt condition which summed with the monocular CMAE found from the colorplus-movement eye.) The "uncertain" reports of apparent movement, obtained on about $25 \%$ of the trials in the other two test conditions, were almost equally divided between positive and negative.

We thus obtained CMAEs from the color-plusmovement eye following an adaptation procedure which was apparently analogous to that of Mikaelian (1975). But we found no consistent evidence, from the achromatic-movement eye, for the movement analogue of the interocular transfer which he reports for the McCollough effect.

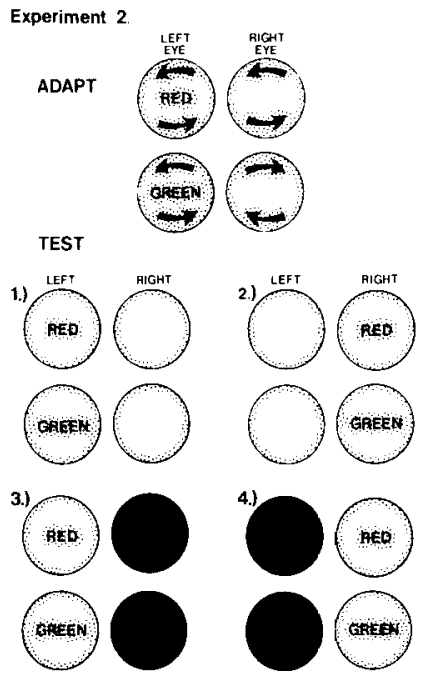

Figure 4. Diagram of the adaptation and test conditions of Experiment 2. Key to the four test conditions: (1) test as adapt, (2) test opposite to adapt, (3) test monocularly color-plus-motion eye, (4) test monocularly motion eye. Other conventions as in Figure 2. See text for further details.

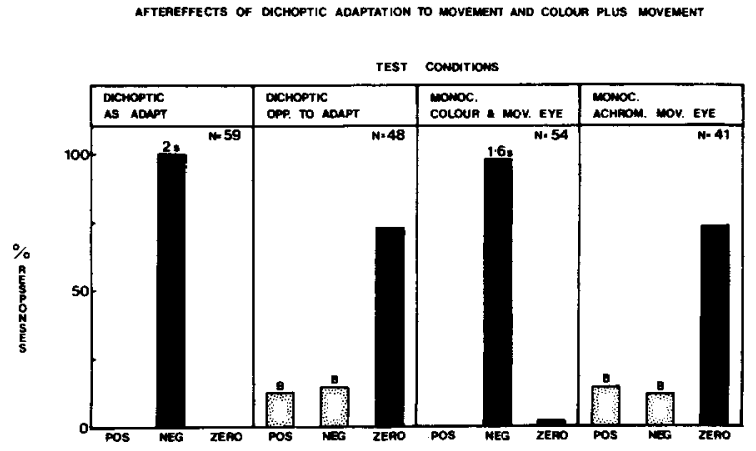

Figure 5. Reports from five subjects of apparent movement of the stationary test field after dichoptic adaptation to alternating achromatic and colored moving fields. Other conventions as in Figure 3.

\section{EXPERIMENT 3}

Before making any theoretical attempt to cope with these results, it seemed worthwhile to repeat the dichoptic experiment, using orientation rather than color as the eliciting dimension for CMAEs.

It was hoped that this experiment would throw light on the binocularity of color-contingent AEs, as well as providing a quantitative basis for comparison with the previous experiments.

\section{Procedure}

The apparatus was as described for Experiment 1, except that the red and green filters in the slide projectors were replaced with orthogonally oriented gratings. The adapting and test conditions are shown schematically in Figure 6. Beam splitters were used for both eyes during adaptation, with their unwanted views occluded with black card. So a subject might adapt to a $45^{\circ}$ left tilted grating (left eye) plus center $\mathrm{CW}$, surround $\mathrm{CCW}$ movement (right eye), alternating with a $45^{\circ}$ right tilted grat- 


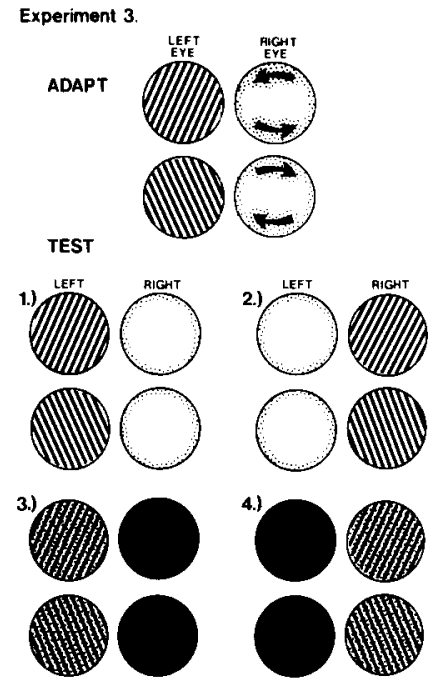

Figure 6. Diagram of adaptation and test conditions of Experiment 3. Key to the four test conditions: (1) test as adapt, (2) test opposite to adapt, (3) test monocularly grating eye, (4) test monocularly motion eye. Other conventions as Figure 2. See text for further details.

ing (left eye) plus center $\mathrm{CCW}$, surround $\mathrm{CW}$ movement (right eye).

Four test conditions were used: test as adapt; test with grating and test field reversed to the eyes; test monocularly grating eye and movement eye separately by mixing the grating and test field in the appropriate beam splitter. Six subjects, including some used in previous experiments, adapted for $20 \mathrm{~min}$.

\section{Results and Discussion}

The results categorized on our previous criteria, are shown in Figure 7. We found evidence for negative CMAEs in all four test conditions, but they could be most easily and strongly elicited in the dichoptic test-as-adapt condition. This test condition consistently yielded CMAEs with a mean duration of $3.5 \mathrm{sec}$. Although up to a third of the responses in the other test conditions were of no apparent movement, such movement, when it occurred, was almost exclusively in the negative direction, though "uncertain" ratings were not uncommon. These CMAEs were reportedly much weaker than those in the dichoptic test-as-adapt condition, and comparable in strength across the three conditions.

This result is clearly consistent with the idea, stemming from previous work on interocular transfer (see, e.g., Campbell \& Maffei, 1971; Wade, 1976) that, in normals, the mechanisms underlying adaptation to tilt and to movement both have a binocular component. The importance of this result lies in its contrast with the finding obtained when MAEs are made contingent on color. Orientation-contingent MAEs show interocular transfer (Potts \& Harris, 1975), and can be evoked in every test condition that we have used; color-contingent MAEs, on the other hand, can apparently be evoked, after either dichoptic or monocular adaptation, only if the eye which has seen color during adaptation also sees color during the test procedure.

It is true that a recent paper by Favreau (1978) has reported interocular transfer of color-contingent movement aftereffects which were positive. In an experiment of our own, which tried to replicate her findings, no evidence was found for such positive effects or for the effect of order of eye testing on which she claims aftereffects from either eye depend. Her results may have arisen because each subject received very few test presentations. We believe that interference from simple adaptation from the last adapting direction of motion, together with the unreliability which we found to be associated with initial test presentations, may have led her to inappropriate conclusions.

It is worthwhile, at this point, to compare the results shown in Figures 3 and 7, in which color and orientation, respectively, were the eliciting stimuli for CMAEs. In two of the four test conditions-dichoptic "as adapt," and monocular tilt or color eye-the results are very similar. In the former condition, for both tilt and color, negative CMAEs of several seconds' mean duration were reported; in the latter condition, although there was no apparent movement on some presentations, when it was reported, its direction was consistently negative. In contrast, the dichoptic opposite-to-adapt and monocular-motion-eye conditions produced different results for color and tilt. For color, both conditions yielded few reports of apparent movement, which were almost equally divided between negative and positive (i.e., no evidence for CMAEs). For tilt, on the other hand, negative CMAEs were regularly reported, although some trials produced reports of no apparent movement. The comparison shows that there was no problem, in principle, in obtaining CMAEs in all test conditions in our apparatus. Notice that with the tilt-contingent experiment, the distribution of responses is similar across the three test conditions which were not identical to the adapting condition. In the color-contingent experiment, on the other hand, this distribution is found only in the monocular-color-eye condition.

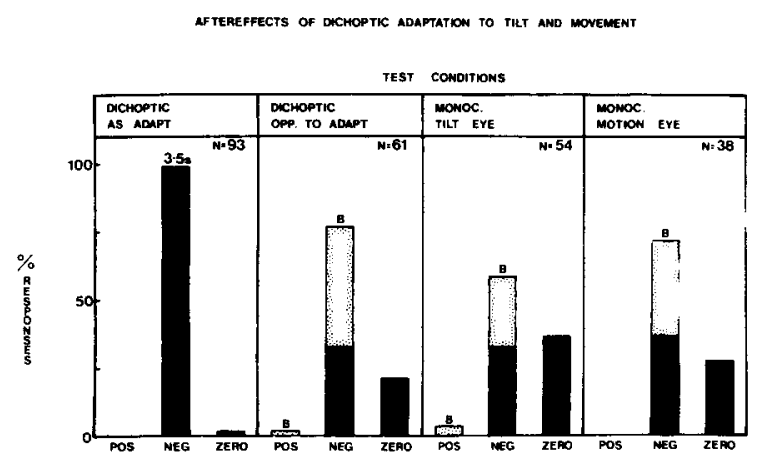

Figure 7. Reports from six subjects of apparent movement of the stationary test field after dichoptic adaptation to alternating tilted gratings and moving fields. Other conventions as for Figure 3. 


\section{GENERAL DISCUSSION}

Our results support the following general conclusions about contingent aftereffects:

(1) The asymmetries of binocular interaction which have been reported for CMAEs (Murch, 1974; Potts \& Harris, 1975) are probably unique to CAEs involving color. This seems up to now to have been a tacit assumption. If it is correct, we predict that any visual dimension which gives interocular transfer of a simple $\mathrm{AE}$, when used as the eliciting dimension will also give CMAEs in all four of the test conditions used here.

(2) Conscious perception of the test field color is not necessary to elicit a color-contingent MAE. This sets an upper limit to the search for the site(s) in the visual system at which this aftereffect originates. A similar conclusion was recently reached by White et al. (1978) from their studies of the effects of binocular suppression on the strength of the McCollough effect.

(3) Our experiments add to the growing body of ' evidence that CAEs involving color can be dichoptically induced, and therefore, in part, have their origin at or after a site at which some kind of binocular interaction has occurred. Our results seem relevant to two proposals which have been made about the nature of this interaction. Of particular interest is our failure to obtain CMAEs via the eye which had adapted only to motion, a failure which replicates that of Murch (1974), whose findings we have extended. This result suggests that the effects of the negative transfer of color information to the contralateral monocular pathway, proposed for the McCollough effect by the Mackays $(1973,1975)$ may be confined to the orientation signaling system. It is not consistent with the Mackays' alternative motion of a central efferent color balancing system. Though it might be argued that our techniques were not sensitive enough to detect weak anomalous effects, the Mackays' (1975) results show that their normal effects in the color eye and their anomalous effects in the pattern eye were of similar strength. Since we could detect normal CMAEs in the color eye in our analogue of their dichoptic experiment, our technique should have detected anomalous effects in the motion eye, if they existed. Secondly, one might ask whether a truly binocular site, such as that proposed by Vidyasagar (1976), could explain our results. Unfortunately, while one could account for the basic finding of dichoptic induction by requiring of such a site that its inputs be simultaneous but not necessarily identical, it is not obvious why it should yield CMAEs only when the color-adapted eye sees color during the test session. The monocular tagging of color information seems to be one requirement of any model which seeks to explain the dichoptic as well as the interocular transfer results.

\section{REFERENCES}

Anstis, S. M. After-effects of seen movement and brightness. PhD thesis, University of Cambridge, 1961.

Campbell, F. W., \& Mafrei, L. The tilt after-effect: A fresh look. Vision Research, 1971, 11, 833-840.

Coltheart, M. Colour-specificity and monocularity in the visual cortex. Vision Research, 1973, 13, 2595-2598.

DAY, R. H., \& Strelow, E. Reduction or disappearance of visual after effect of movement in the absence of patterned surround. Nature, 1971, 230, 55-56.

Favreau, O. E. Interocular transfer of color-contingent motion aftereffects: Positive aftereffects. Vision Research, $1978,18,841-844$.

Favreau, O. E., Emerson, V., \& Corballis, M. C. Motion perception: A color-contingent aftereffect. Science, 1972, 176, 78-79.

Julesz, B. Foundations of cyclopean perception. Chicago: University of Chicago Press, 1971.

LehmkUhle, S. W., \& Fox, R. Effect of binocular rivalry suppression on the motion aftereffect. Vision Research, 1975, 15, 855-859.

Mackay, D. M., \& Mackay, V. Orientation-sensitive after-effects of dichoptically presented colour and form. Nature, 1973, 242, 477-479.

Mackay, D. M., \& Mackay, V. Dichoptic induction of McCollough-type effects. Quarterly Journal of Experimental Psychology, 1975, 27, 225-233.

MaYhew, J. E. W., \& ANSTis, S. M. Movement aftereffects contingent on color, intensity, and pattern. Perception \& Psychophysics, 1972, 12, 77-85.

McCollough, C. Color adaptation of edge-detectors in the human visual system. Science, 1965, 149, 1115-1116.

Mikaelian, H. H. Interocular generalization of orientation specific color aftereffects. Vision Research, 1975, 15, 661-664.

Moulden, B. After-effects. PhD thesis, University of Reading, 1974.

Murch, G. M. Color contingent motion aftereffects-single or multiple levels of processing? Vision Research, 1974, 14, 1181-1184.

Over, R., Long, N., \& Lovegrove, W. Absence of binocular interaction between spatial and color attributes of visual stimuli. Perception \& Psychophysics, 1973, 13, 534-540.

PotTs, M. J., \& Harris, J. P. Movement aftereffects contingent on the pattern or colour of a stationary surround. Vision Research, 1975, 15, 1225-1230.

VIDYASAgAR, T. R. Orientation specific colour adaptation at a binocular site. Nature, 1976, 261, 39-40.

WADE, N. J. On the interocular transfer of the movement aftereffect in individuals with and without normal binocular vision. Perception, 1976, 5, 113-118.

WADE, N. J., \& Wenderoth, P. The influence of colour and contour rivalry on the magnitude of the tilt after-effect. Vision Research, 1978, 18, 827-835.

White, K. D., Petry, H. M., Riggs, L. A., \& Miller, J. Binocular interactions during establishment of McCollough effects. Vision Research, 1978, 18, 1201-1215.

(Received for publication July 17, 1978; revision accepted February 12, 1979.) 